\title{
Etudes sur la qualité de l'eau dans l'estuaire du Tage
}

\section{Study of water quality in the Tagus Estuary}

\author{
P.M. Fiadeiro
}

Instituto Hidrográfico - Portugal

\section{Introduction}

L'estuaire du Tage (Fig. 1) est un excellent port naturel sur la côte du Portugal. La ville de Lisbonne, sur la rive droite, concentre la plupart des activités portuaires. L'estuaire reçoit les eaux de rejets non traitées des deux rives, des émissaires urbains et industriels, et les eaux de ruissellement des zones agricoles. L'estuaire est aussi un domaine de la pêche, des loisirs et un refuge pour les oiseaux migrateurs venus d'Europe en hiver.

Du fait de son importance pour le développement de Lisbonne, l'estuaire a fait l'objet de plusieurs études. Les autorités portuaires ont étudié la sédimentation depuis le début du $19^{\mathrm{e}}$ siècle ; plus récemment d'autres études concernent le prisme à marée [1], la stratification et les courants. Quelques exemples de profils verticaux de salinité sont présenté sur la figure 2. Enfin, a été construit au "Laboratório Nacional de Engenharia Civil" un modèle hydraulique à l'échelle horizontale de 1:500 et verticale de 1:70 pour l'étude des courants de marée et la sédimentation.

D'autres études [2], [3] concernant la qualité de l'eau et pour le tracé des égouts de Lisbonne ont conduit à la conclusion que l'estuaire était capable d'assimiler les charges des effluents sans dégradation significative de la qualité de l'eau. Plus tard est apparue une certaine dégradation de la qualité de l'eau [4].

Ensuite, d'autres études ont été reprises en 1976 de façon à déterminer une méthode à la fois plus efficace et plus économique pour améliorer la qualité de l'eau [5]. Une partie de ces études concernait la dynamique estuarienne.
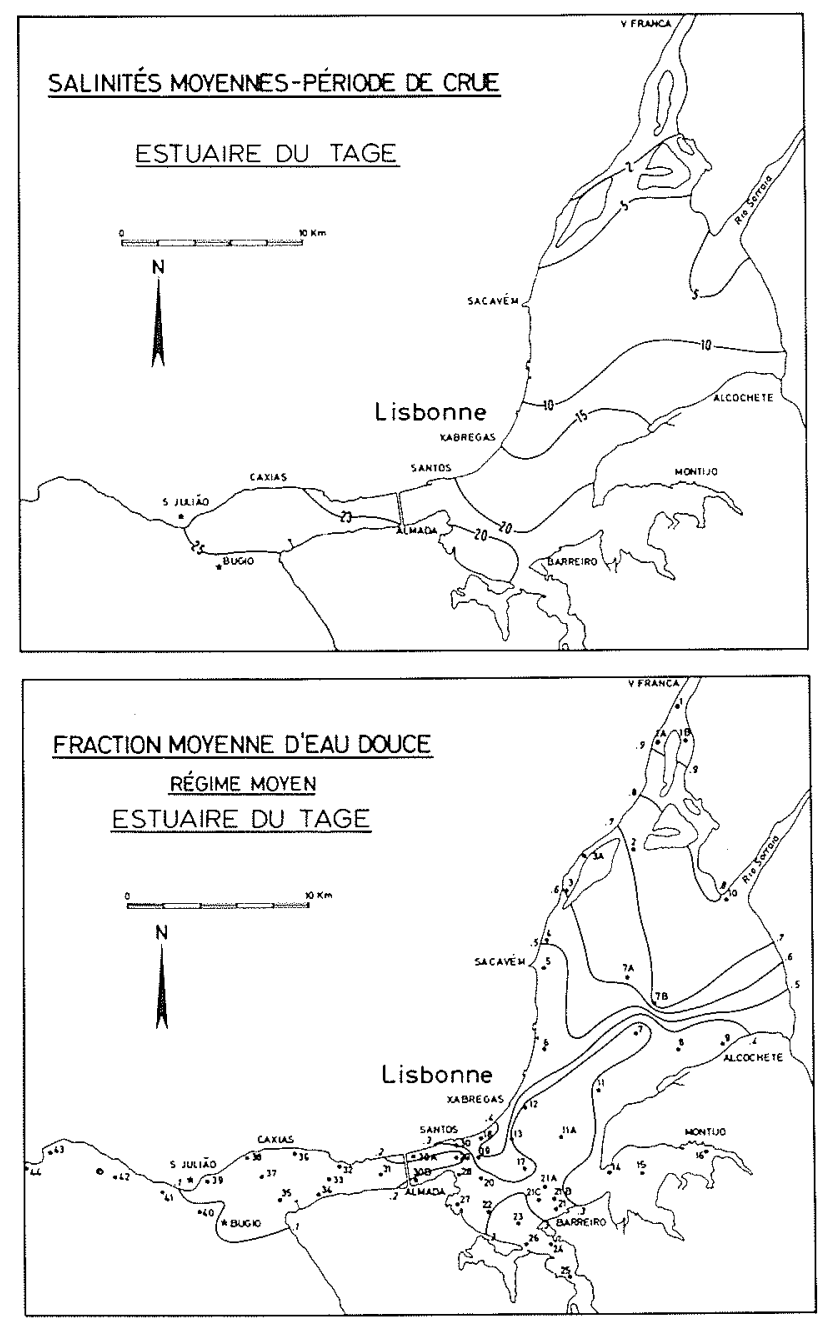

Figure 1 

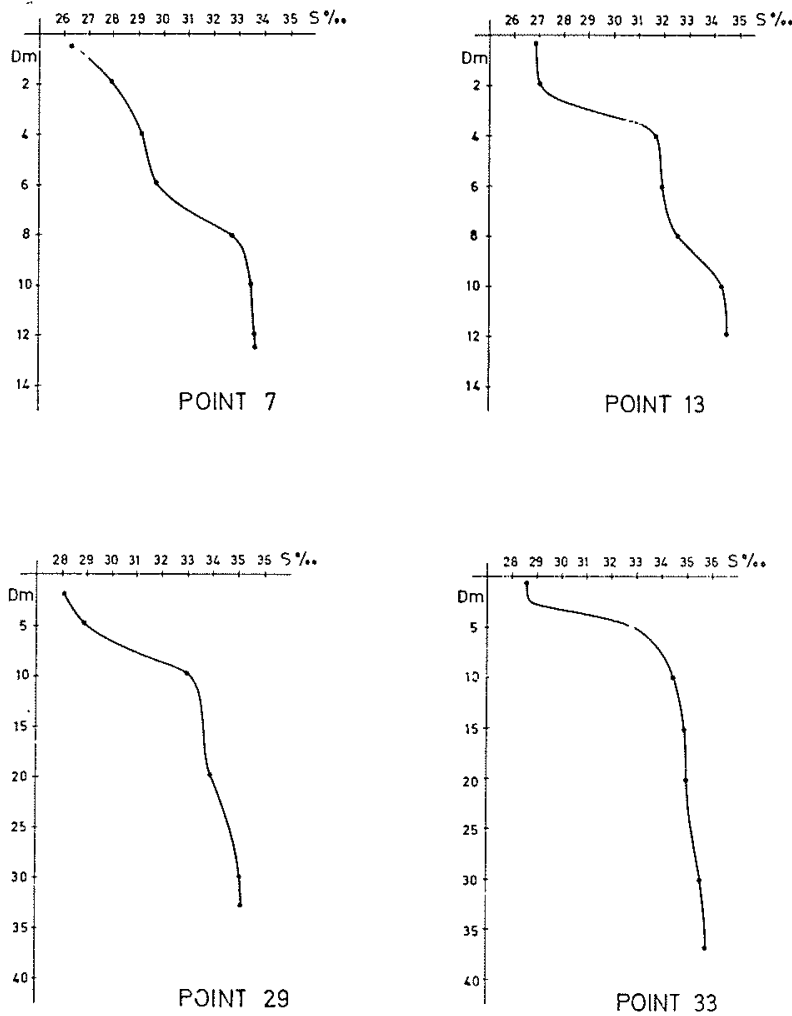

Figure 2 - Profils verticaux de salinité. Estuaire du Tage (Morte eau-étiage)

\section{Les caractéristiques de l'estuaire}

Les mesures de propagation de la marée situent sa limite amont à environ $80 \mathrm{~km}$ de l'embouchure. Le volume du prisme de marée est de l'ordre de $7.10^{8} \mathrm{~m}^{3}$.

Le temps de résidence de l'eau douce, défini par le temps nécessaire pour le renouvellement total du volume d'eau douce par le débit fluvial a été calculé [6], [7], à partir des profils de salinité qui correspondaient aux régimes hydrologiques moyens d'étiage et de crue (Tableau I).

\begin{tabular}{|c|c|c|}
\hline Tableau I & \multicolumn{2}{c|}{ Temps de résidence de l'eau douce } \\
\hline Débit fluvial & Volume total & Temps de résidence \\
\hline moyen $\left(400 \mathrm{~m}^{3} / \mathrm{s}\right)$ & $6,1 \times 10^{8} \mathrm{~m}^{3}$ & 18 jours \\
\hline étiage $\left(100 \mathrm{~m}^{3} / \mathrm{s}\right)$ & $4,2 \times 10^{8} \mathrm{~m}^{3}$ & 49 jours \\
\hline crue $\left(750 \mathrm{~m}^{3} / \mathrm{s}\right)$ & $8,1 \times 10^{8} \mathrm{~m}^{3}$ & 12 jours \\
\hline
\end{tabular}

\section{Caractéristiques de l'écoulement}

La classification de l'estuaire selon le critère de Pritchard [8], basé sur le rapport entre le prisme à marée et le débit fluvial, conduit à un écoulement à deux couches avec mélange vertical pour le régime moyen.
Dans le même ordre d'idée, en utilisant le nombre d'estuaire et le nombre de Froude qui caractérise la contribution des forces inertielles (courants de flot) et des forces de gravité (salinités), le calcul a été fait pour trois points de l'estuaire.

La stratification diminue vers l'amont ; elle est légère surtout aux points 7 et $15 \mathrm{~A}$.

La méthode, qui semble être la plus correcte et la plus complète [9] pour caractériser l'estuaire, utilise la relation entre l'indice de stratification et l'indice de circulation :

- L'indice de stratification (IS) est le rapport entre la différence de salinités entre la surface et le fond et la salinité moyenne de la section droite de l'estuaire ;

- L'indice de circulation (IC) est le rapport entre la vitesse résiduelle en surface et celle due au débit fluvial à travers la même section.

Selon cette classification, l'estuaire du Tage est partiellement mélangé. Les processus diffusifs et advectifs contribuent sensiblement au flux de sel vers l'amont, avec une stratification appréciable. Les différentes valeurs du rapport entre les processus diffusifs et advectifs, appelées fraction diffusive, sont représentées sur la figure 3. Plus récemment, les calculs ont été repris avec de nouvelles mesures. Les résultats sont aussi portés sur la figure 3 .

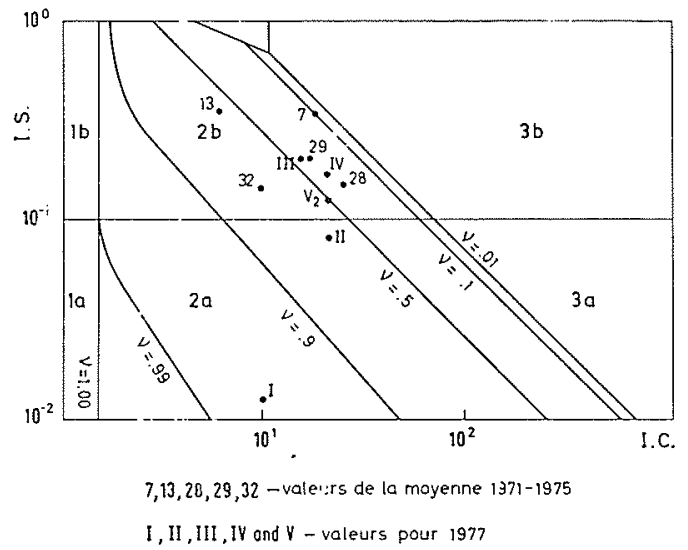

Figure 3 - Diagramme de Hansen et Rattray. Estuaire du Tage.

Le calcul des vitesses résiduelles conduit aux résultats de la figure 4.

On peut partager la vitesse résiduelle en quatre composantes :

$$
u(y, z)=u_{1}(z)+u_{2}+u_{3}(y)+u_{4}
$$

$u_{1}(z)$ : courant de densité dû aux variations de densité au long de l'estuaire,

$u_{2}$ : courant dû au débit fluvial

$u_{3}(y)$ : composante due à la forme de l'estuaire

$u_{4}$ : composante due aux inégalités de la marée.

De la même façon on peut partager la salinité

$$
S(y, z)=S 1(z)+S 2+S 3(y)
$$


et obtenir le débit de sel résiduel

$$
\begin{aligned}
\int_{0}^{B} \int_{0}^{D} u S d y d z=B \int_{0}^{D} u_{1} S_{1} d z & +A u_{2} S_{2}+ \\
& +D \int_{0}^{B} u_{3} S_{3} d y
\end{aligned}
$$

Le premier et le deuxième terme représentent le transport advectif respectivement vers l'amont par courant de densité et vers l'aval par le débit fluvial ; désignonsles respectivement par:

$$
\underline{T A}=B \int_{0}^{B} u S d z \text { et } \underline{T R}=-A_{2} S_{2}=R \bar{S}
$$

avec $R$ débit fluvial et $\bar{S}$ salinité moyenne.

Le troisième terme, qui provient de la corrélation entre les variations latérales de courant et de salinité, peut être considéré comme une partie du terme de diffusion turbulent $T D$

$$
\underline{T D}=D \int_{0}^{B} u_{3} S_{3} d y-A K_{x} \frac{\partial \bar{S}}{\partial x}
$$

ou, si on introduit le coefficient modifié $K_{x}^{*}$,

$$
\underline{T D}=-A K_{x}^{*} \frac{\partial \bar{S}}{\partial x}
$$

Alors, à partir de l'équation de continuité stationnaire dus sel

$$
\underline{T R}=\underline{T A}+\underline{T D}
$$

Le transport de sel par le débit fluvial est donc équilibré par l'addition d'un terme de transport advectif par le courant de densité et d'un terme diffusif. On peut donc définir la fraction diffusive :

$$
D=T D /(T A+T D)
$$

et en se plaçant dans l'état stationnaire :

$$
D=(T R-T A) / T R
$$

Les résultats des calculs, dans le cas du Tage, sont présentés dans le tableau II où les valeurs graphiques sont comparées à celles calculées analytiquement [10].

\begin{tabular}{|l|c|c|c|c|c|}
\hline Tableau II & \multicolumn{5}{|c|}{ Valeur de la fraction diffusive } \\
\hline $\begin{array}{c}\text { Points } \\
\text { (voir figure 1) }\end{array}$ & $I_{(2)}$ & $I_{(1)}$ & $I_{(2)}$ & $I V_{(1)}$ & $V_{(1)}$ \\
\hline$D_{\text {graphique }}$ & $0,9-0,99$ & $0,5-0,9$ & $0,1-0,5$ & $0,1-0,5$ & $0,5-0,9$ \\
\hline$D_{\text {analytique }}$ & 0,999 & 0,905 & 0,245 & 0,387 & 0,742 \\
\hline (1) 22/06/1977;(2) $23 / 06 / 1977$ \\
\hline
\end{tabular}

La salinité moyenne est plus élevée près de la rive gauche, ce qui permet de suggérer l'existence de chenaux de flot et de jusant. La circulation résiduelle (Fig. 4) appuie cette hypothèse. Ce fait pouvant être dû à la force de Coriolis, on a calculé le nombre de Rossby, rapport entre les forces d'inertie totale et la force de Coriolis et aussi le rapport entre la durée du demi-jour pendulaire et la période de la marée. Pour le Tage il vaut 1,54 ce qui correspond à une relative importance de la force de Coriolis.

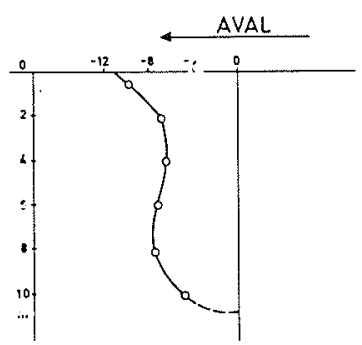

POINT I $(23 ; 6 / 77)$

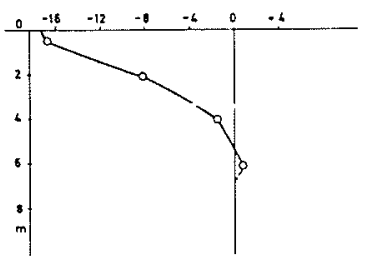

POINT $111(23 / 6 / 77)$

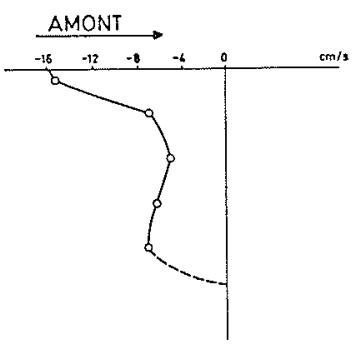

POINT II $(22 / 6 / 77)$

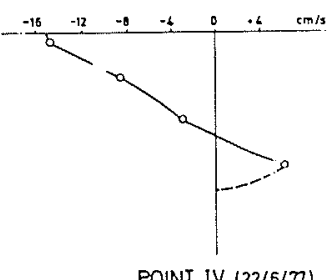

POINT IV $(22 / 6 / 7)$

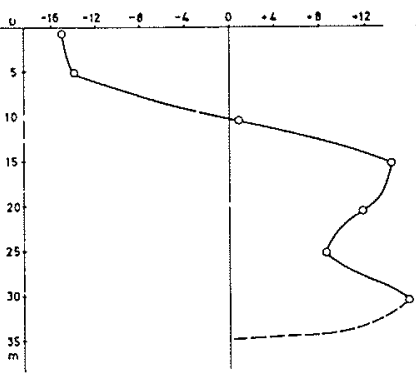

POINT V $(22 / 6 / 77)$

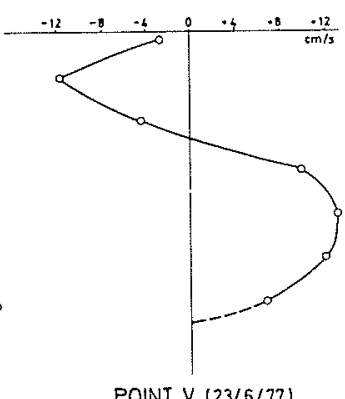

POINT V $(23 / 6 / 77)$

Figure 4 - Courants résiduels. Estuaire du Tage.

Un facteur important est la géométrie de l'estuaire qui, à partir de quelques particularités de l'écoulement, peut dans certains estuaires, masquer l'influence de la force de Coriolis.

\section{Corrélations des paramètres de la qualité de l'eau}

Des corrélations ont été tentées avec les données historiques disponibles [4] pour la marée et pour le débit fluvial, avec l'oxygène dissous, phosphates, nitrates, nitrites, silicates, $p H$ et bactéries fécales. Les données ont été ajustées par régression linéaire simple, sauf pour les bactéries pour lesquelles on a utilisé la régression loglinéaire.

Les données de juin 1977 ont été utilisées pour les calculs de ces corrélations, mais l'accord ne s'est manifesté pour le débit fluvial, que dans un nombre limité de cas. De façon générale, le niveau d'oxygène dissous a diminué nettement; les valeurs de quelques paramètres ont augmenté par rapport à la situation antérieure, révélant une augmentation de pollution.

\section{Coefficients de dispersion}

Plusieurs études sur les coefficients de dispersion sont connues ; mais les estuaires et surtout le Tage, ne 
sont pas des chenaux simples à géométrie régulière pour lesquels la plupart des auteurs obtiennent des formules simplifiées, mais pas toujours réalistes. Dans cette optique ont été calculés les coefficients de dispersion longitudinal $K_{x}$ et vertical $K_{z}$ ainsi que la viscosité turbulente verticale $N_{z}$ avec comme but de considérer un modèle préliminaire bidimensionnel dans le sens vertical. Le tableau III rassemble les résultats.

\begin{tabular}{|c|r|r|r|r|r|}
\hline \multicolumn{5}{|c|}{ Tableau III } \\
\hline $\begin{array}{c}\text { Points } \\
\text { (voir figure 1) }\end{array}$ & ${ }^{\prime}(2)$ & $I_{(1)}$ & III $_{(2)}$ & $I_{(1)}$ & $V_{(1)}$ \\
\hline$K_{x} \times 10^{6}\left(\mathrm{~cm}^{2} / \mathrm{s}\right)$ & 0,18 & 4,84 & 5,06 & 4,76 & 7,57 \\
\hline $\mathrm{Kz}\left(\mathrm{cm}^{2}\right)$ & 8,35 & 18,09 & 4,37 & 3,40 & 20,14 \\
\hline $\mathrm{Nz}\left(\mathrm{cm}^{2} / \mathrm{s}\right)$ & 32,25 & 32,48 & 19,35 & 14,94 & 48,12 \\
\hline (1) $22 / 06 / 1977:(2) 23 / 06 / 1977$ \\
\hline
\end{tabular}

\section{Conclusions}

L'importance du prisme à marée dans le Tage par rapport au débit fluvial, conduit à un écoulement à deux couches où les processus advectifs sont responsables du flux de sel vers l'amont avec une stratification appréciable.

Les tendances à long terme montrent que l'oxygène dissous ne pose pas de problèmes pour le moment ; mais il apparait une contamination importante en bactéries fécales dans la partie aval de l'estuaire. Compte-tenu du long temps de résidence de l'eau douce, en régime d'étiage la capacité d'assimilation de l'estuaire est à la limite.

Les corrélations des paramètres de qualité de l'eau avec le débit fluvial, permettent de considérer le comportement des polluants de façon semblable à l'eau douce et donc à employer pour ceux-ci les coefficients de dispersion valables pour le sel.

La répartition des écoulements résiduels dans l'estuaire se manifeste par un écoulement dirigé vers l'amont près $\mathrm{d} u$ fond rive gauche (le chenal de flot) et un écou- lement toujours dirigé vers l'aval rive droite (le chenal de jusant). Les coefficients de dispersion sont du même ordre de grandeur que ceux qu'on rencontre dans la littérature.

\section{Remerciements}

Ce travail a été subventionné par la "Commissão Nacional do Ambiente". Je tiens à remercier le Directeur Monsieur D. Palmer, du Ministère de l'Environnement d'Ontario, Canada, pour son encouragement et fructueuses discussions, ainsi que le Docteur R. Bonnefille d'Electricité de France pour ses commentaires et sa révision du manuscrit.

\section{Références}

[1] LEMOS P.F. - Estuário do Tejo. AGPL, Lisboa, 1972.

[2] OLIVEIRA E.A. - Estogos de Lisboa. Estudos de Anteprojectos, Câmara Municipal de Lisboa, Lisboa, 1941.

[3] COSTAT P.C. - Poluição do Estuário do Tejo. Técnica, Lisboa, 1957.

[4] ATAIDE J. - Pollution Data of the Tagus Estuary and Coastal Waters, 1971-1975 Pilot Study for CCMS of NATO, Experts Meeting, Ostend 9-13 June 1975, 1975.

[5] PALMER M.D., FIADEIRO P., DEL NEGRO M. - Environmental Study of the Tejo estuary. First progress report, Comissão Nacional do Ambiente, Lisbon, November 1977.

[6] KETCHUM B.H. - Hydrographic factors involved in the dispersion of pollutants introduced into tidal waters. Jour. Boston Soc. Civil Eng., n ${ }^{\circ}$ 37, 1950, pp. 296-314.

[7] ALLEN G.P. - Etude des processus sédimentaires dans lestuaire de la Gironde. Thèse de doctorat d'Etat ès Sciences Naturelles, Univ. de Bordeaux I, 1972.

[8] PRITCHARD D.W. - Estuarine circulation patterns, Proc. Amer. Soc. Civil Eng. vol. 81, $\mathrm{n}^{\circ} 717,1955$.

[9] HANSEN D.V., RATTRAY Jr. M. - New dimensions in estuary classification. Limn. and Oceanog. vol. II, $\mathrm{n}^{\circ}$ 3, 1966, pp. 319-326.

[10] BONDEN K.F., GILLIGAN R.H. - Caracteristic features of estuarine circulation as represented in the Mersey Estuary, Limn, and Oceanog., vol. 16, $\mathrm{n}^{\circ} 3,1971$, pp. 490-502. 